\title{
A study of correlation between the oscillation amplitude and stellar parameters of $\delta$ Scuti stars in open clusters
}

\section{Toward selection rules for $\delta$ Scuti star oscillations}

\author{
J.-C. Suárez ${ }^{1}$, E. Michel ${ }^{1}$, F. Pérez Hernández ${ }^{3,4}$, Y. Lebreton ${ }^{2}$, Z. P. Li ${ }^{5}$, and L. Fox Machado ${ }^{3}$ \\ 1 LESIA, Observatoire de Paris-Meudon, FRE 2461, France \\ e-mail: Eric.Michel@obspm.fr \\ 2 GEPI, Observatoire de Paris-Meudon, FRE 2459, France \\ e-mail: Yveline.Lebreton@obspm.fr \\ ${ }^{3}$ Instituto de Astrofísica de Canarias (IAC), 38200 La Laguna, Tenerife, Spain \\ e-mail: fph@ll.iac.es; lfox@ll.iac.es \\ 4 Departamento de Astrofísica, Universidad de La Laguna, Tenerife, Spain \\ 5 National Astronomical Observatories, Chinese Academy of Sciences, 100012 Beijing, PR China \\ e-mail: lizhi@bao.ac.cn
}

Received 6 March 2002 / Accepted 3 April 2002

\begin{abstract}
In the present work, we study correlations between stellar fundamental parameters and the oscillation amplitude for $\delta$ Scuti stars. We present this study for a sample of 17 selected $\delta$ Scuti stars belonging to 5 young open clusters. Taking advantage of properties of $\delta$ Scuti stars in clusters, we correct the photometric parameters of our objects for the effects of fast rotation. We confirm the benefit of applying such corrections in this kind of studies. In addition, the technique used for this correction allows us to obtain an estimate of stellar parameters such as the angle of inclination and the rotation rate, usually not accessible. A significant correlation between the parameter $i^{\prime}$ (estimation of the angle of inclination of the star) and the oscillation amplitude is found. A discussion and interpretation of these a priori surprising results is proposed, in terms of a possible selection rule for oscillation modes of $\delta$ Scuti stars.
\end{abstract}

Key words. stars: variables: $\delta$ Sct - stars: rotation - stars: statistics - stars: oscillations - stars: fundamental parameters galaxies: star clusters

\section{Introduction}

The $\delta$ Scuti stars are variables located in the lower part of the Cepheid instability strip with spectral types from A2 to F0. These pulsators, when belonging to the Main Sequence, seem particularly suitable for determining the extent of the convective core and internal rotation rate, and thereby for probing poorly understood hydrodynamical processes occurring in deep stellar interiors. Within the last decade great efforts have been made in developing the seismology of $\delta$ Scuti stars (Breger 2000; Handler 2000). However, several aspects of the pulsating behavior of these stars (e.g. the number and the determination of the excited modes) within the instability strip are not completely understood.

Several statistical works investigated potential correlations between the oscillation amplitude and different stellar parameters. Particularly, a correlation between the amplitude, period and absolute magnitude of low amplitude $\delta$ Scuti stars has been established (Antonello et al. 1981). In a preliminary work

Send offprint requests to: J.-C. Suárez,

e-mail: JuanCarlos.Suarez@obspm.fr for $\delta$ Scuti stars in the Praesepe cluster, Suárez et al. (2001) have suggested the importance of taking into account the effect of fast rotation as proposed by Michel et al. (1999) in such studies.

Here we extend this work to 5 open clusters, in order to estimate the impact of the mentioned technique in the studies of correlation. In addition, these tools allow us to obtain a rough estimate of several stellar parameters like the angle of inclination of the star or the rotation rate, that are not usually accessible. We present a study of single correlations between the parameters mentioned before and the oscillation amplitude. For values indicating a reasonable degree of correlation, we also search for a possible functional dependence.

This paper is organized as follows: in Sect. 2 we present the observational material and the main characteristics of the selected open clusters. Details on the computation of models are given in Sect. 3. In Sect. 4, we introduce the stellar parameters corrected for the effect of fast rotation. Section 5 is dedicated to describe the technique of correlation and the analysis of the results. Finally, we present our conclusions in Sect. 6. 
Table 1. Main characteristics of the 5 open clusters used in this work. Different columns represent the cluster name; distance modulus $(d)$; metallicity (Cols. 3 and 4$)$; reddening $\left(\delta E=E\left(B_{2}-V_{1}\right)\right)$ and age.

\begin{tabular}{lccccc}
\hline \hline Cluster & $\begin{array}{c}d \\
(\mathrm{mag})\end{array}$ & {$[M / H]$} & $Z$ & $\begin{array}{c}\delta E \\
(\mathrm{mag})\end{array}$ & $\begin{array}{c}\text { Age } \\
(\mathrm{Myr})\end{array}$ \\
\hline Praesepe & 6.28 & 0.170 & 0.025 & 0.0 & 650 \\
Pleiades & 5.50 & -0.112 & 0.014 & 0.05 & 130 \\
Hyades & 3.33 & 0.143 & 0.012 & 0.0 & 600 \\
Coma Ber & 4.70 & -0.048 & 0.016 & 0.0 & 430 \\
$\alpha$-Persei & 6.23 & -0.05 & 0.016 & 0.09 & 90 \\
\hline
\end{tabular}

Table 2. Indivual parallaxes for selected $\delta$ Scuti stars of Hyades. $\pi$ represents the parallax in miliarcseconds and $(m-M)_{v}$ is the resulting distance modulus.

\begin{tabular}{ccc}
\hline \hline Star & $\begin{array}{c}\pi \\
(\mathrm{mas})\end{array}$ & $\begin{array}{c}(m-M)_{v} \\
(\mathrm{mag})\end{array}$ \\
\hline HD 27397 & $22.38 \pm 0.37$ & $3.25 \pm 0.05$ \\
HD 27459 & $20.87 \pm 0.32$ & $3.40 \pm 0.10$ \\
HD 27628 & $22.30 \pm 0.36$ & $3.25 \pm 0.05$ \\
\hline
\end{tabular}

\section{Observational data}

The oscillation amplitude (hereafter $A_{\text {osc }}$ ), classically, is taken to be the highest peak-to-peak measurement in the observed light curve. Values for $A_{\text {osc }}$ used here are taken from $\mathrm{Li} \&$ Michel (1999) and references therein. The interpretation of this parameter is not straightforward; it can be considered as a maximum constructive interference of the observed modes. Thus, in such an interpretation, and for a given angle of inclination, it can be taken as an indicator of the energy of these modes. With such an estimate, it is evident that short time series might induce an underestimation of the amplitude, especially in presence of beating phenomena due to close frequencies. In order to take this effect into account, we associate with each amplitude estimation a weight corresponding to the length of the time series. In Table 5 we list such values where number 1 corresponds to effective time series shorter than 4 hours; 2 , between 4 and 20 hours; 3, between 20 and 200 hours, and finally 4 corresponds to time series longer than 200 hours. The five young open clusters considered are: Praesepe, Pleiades, Hyades, Coma Ber and $\alpha$-Persei, with ages between 90 and 700 Myr. Detailed information concerning metallicity, age, distance modulus and reddening are listed in Table 1. Apparent magnitudes and colour indexes are taken from Rufener's cataloge (Rufener 1999).

For $\alpha$-Persei, the estimation of the global metallicity $[M / H]$ by spectroscopic measurements is taken from Pinsonneault et al. (1998). For the other four clusters, we used metallicities derived by M. Grenon from Geneva photometry of single stars with spectral type in the range F4-K3 (Grenon 2000; Robichon et al. 1999). To correct the visual absorption due to interstellar matter, the relation

$A_{v}=3.6 E\left(B_{2}-V_{1}\right)$

was used. The intrinsic colours in the Geneva photometric system were obtained from Nicolet (1981).
The distance moduli for Coma $(4.70 \pm 0.04 \mathrm{mag})$ and Praesepe $(6.28 \pm 0.12 \mathrm{mag})$ are derived from mean cluster parallaxes computed using Hipparcos intermediate data (Robichon et al. 1999). In the case of Pleiades, the estimation of the distance modulus is still a subject of discussion. The Hipparcos values $(5.36 \pm 0.06)$ and the one obtained by Pinsonneault et al. (1998), $5.60 \pm 0.06$ mag illustrate the range of values found in the literature (Stello \& Nissen 2001). For the Hyades, we also have access from the literature, to the individual parallaxes of our target $\delta$ Scuti stars (de Bruijne et al. 2001) that are listed in Table 2. For the other stars we use an estimation of $3.33 \pm 0.01$ mag by Perryman et al. (1998) from Hipparcos observations. Finally for $\alpha$-Persei, we used a distance modulus of $6.23 \pm 0.06$ from Pinsonneault et al. (1998).

The projected velocity $(V \sin i)$ values listed in Table 5 are taken from Uesugi \& Fukuda (1982).

\section{Stellar models and isochrones}

The stellar evolutive models have been computed using the evolution code CESAM (Morel 1997). We consider input physics appropriate for the mass range covered by $\delta$ Scuti stars. Metallicity values $(Z)$ used to compute models are deduced from $[M / H]$ assuming $Y_{\mathrm{pr}}=0.235$ and $Z_{\mathrm{pr}}=0$ as helium and heavy element primordial concentrations, and an enrichment ratio of $\Delta Y / \Delta Z=2$. We work with OPAL radiative opacity tables (Iglesias \& Rogers 1996). For the atmosphere reconstruction, Eddington's $T(\tau)$ law is considered. Convection is described according to the classical mixing-length theory, with a solar calibrated mixing length parameter $l_{\mathrm{m}}=1.61 \mathrm{H} p$, where $\mathrm{H} p$ is the local pressure scale-height. For all the models, we take into account an overshooting of the mixed convective core over a distance $\alpha_{\mathrm{ov}}=0.2 \mathrm{Hp}$ following the prescription of Schaller et al. (1992).

Sets of evolutionary sequences were computed for masses between $1 M_{\odot}$ and $5 M_{\odot}$ from the zero-age Main Sequence to the subgiant branch. With representative sequences for each cluster, we obtain the corresponding isochrones using the Geneva isochrone program. These isochrones were then transformed into a $M_{v}-\left(B_{2}-V_{1}\right)$ diagram using the calibration of Kunzli et al. (1997) for $\left(B_{2}-V_{1}\right)$ and the calibration of Schmidt-Kaler (1982) for $M_{v}$.

In Fig. 1 we present colour-magnitude (hereafter CM) diagrams with the observed data for each cluster. Continuous lines correspond to their associated isochrones, given the distances and ages listed in Table 1. Potential binarity and the effect of fast rotation discussed in Sect. 4 are expected to induce systematic shifts toward higher luminosity and lower effective temperature, compared to single non-rotating stars. Thus, in order to compensate for both effects, the fit of isochrones has been made adjusting them to the bottom envelope of the cluster in the CM diagrams.

In the case of Pleiades, we use our best fit corresponding to a distance modulus of $5.5 \mathrm{mag}$, which is approximately half way between the main sequence fitting result and Hipparcos distance (see Sect. 2). The corresponding age found is $130 \mathrm{Myr}$. 

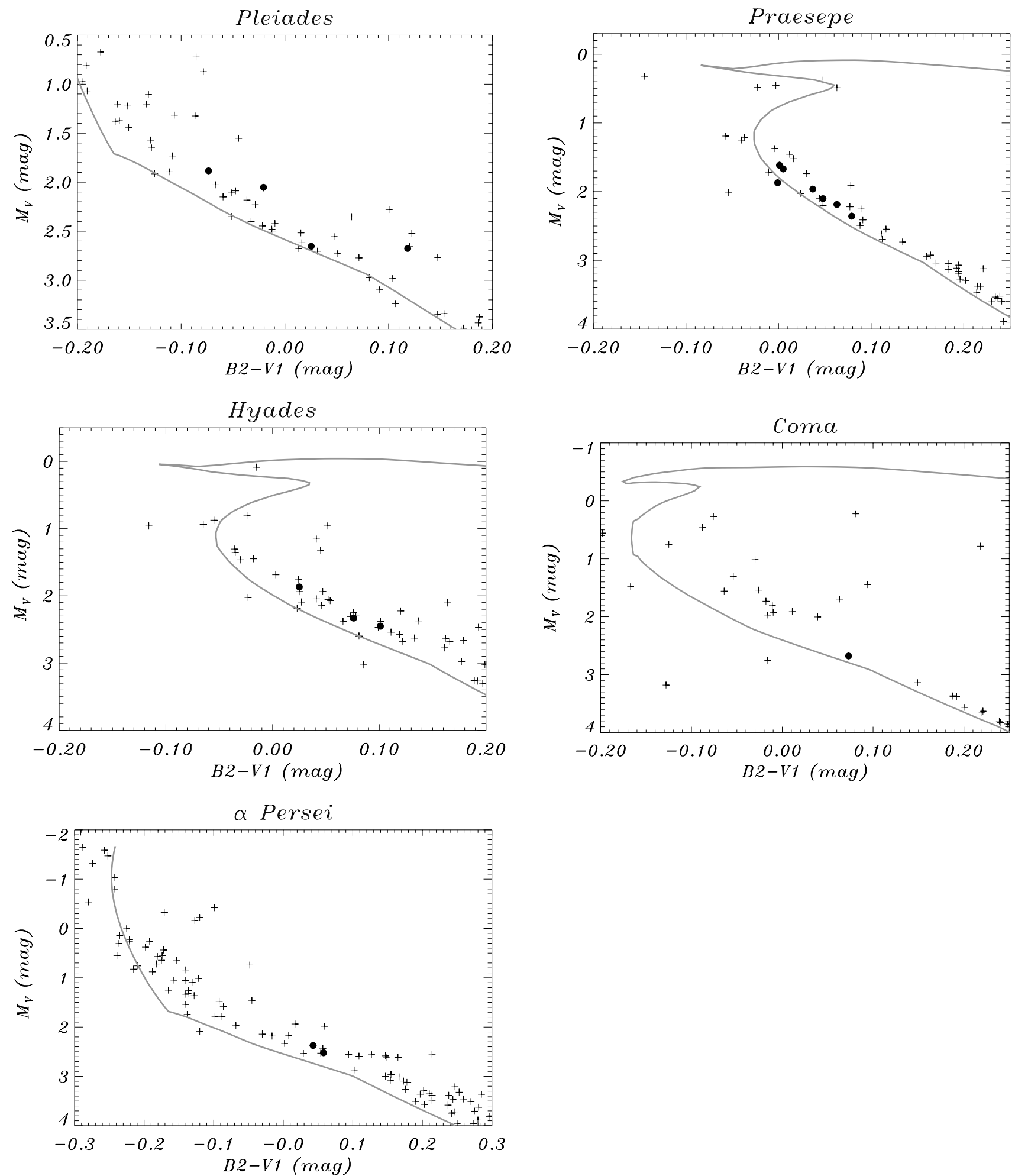

Fig. 1. Colour-Magnitude diagrams for the selected 5 open clusters. Filled circles and crosses represent respectively the observed $\delta$ Scuti and other stars belonging to each cluster. The correction for distance and reddening is taken into account as mentioned in the text (see Sect. 2).

\section{Stellar parameters corrected for the effect of fast rotation}

We will not enter here into the details of the method, already described in Pérez Hernández et al. (1999). Following this paper, we apply the method to selected $\delta$ Scuti stars for each of our five clusters. In Fig. 2 we illustrate such corrections for the five clusters. For a given $V \sin i$, segments represent the potential position of the star, in the absence of rotation, on the $\mathrm{CM}$ diagram. The position of the non-rotating copartners varies 

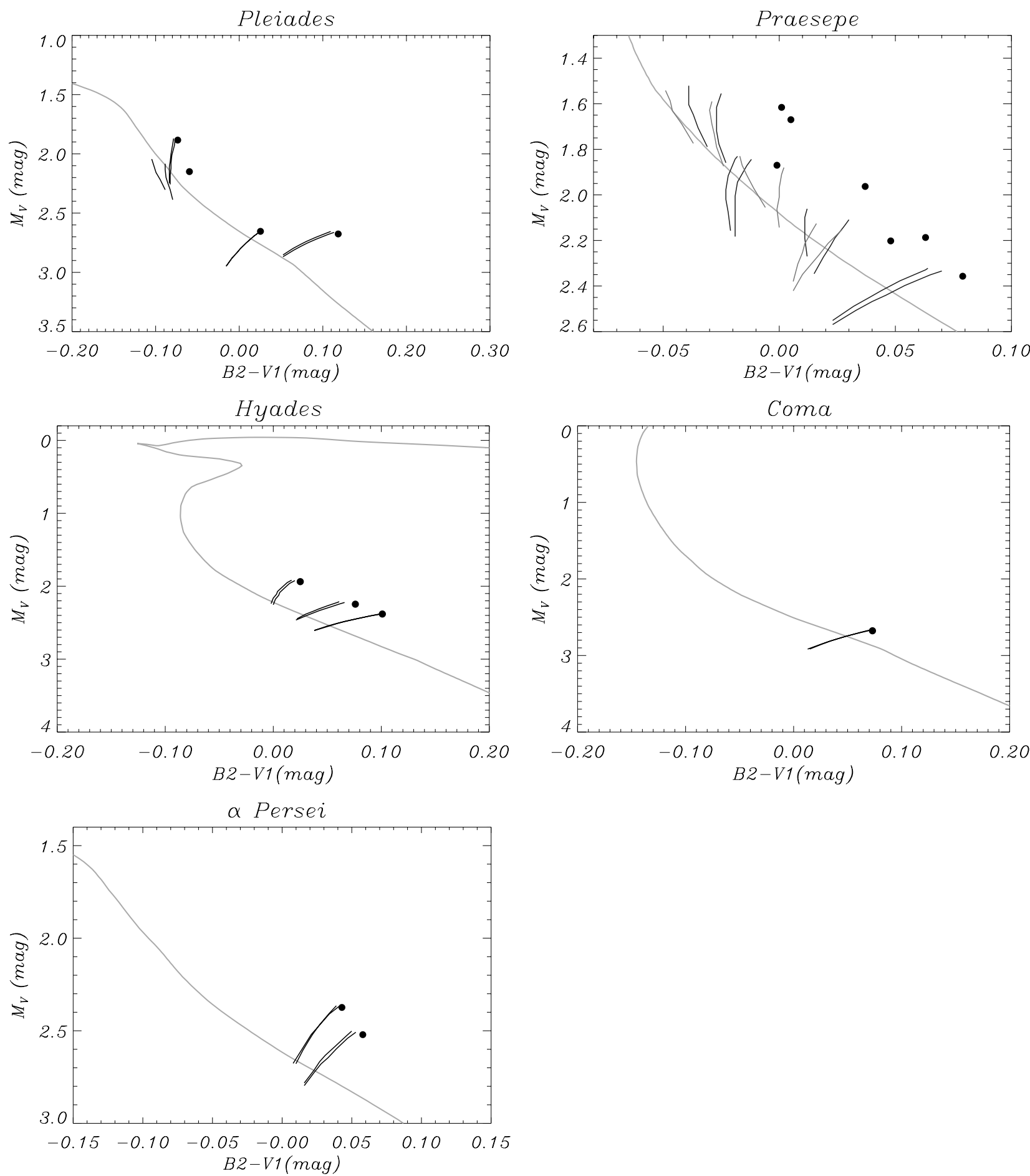

Fig. 2. Colour-Magnitude diagrams for the selected 5 open clusters. Filled circles represent the observed $\delta$ Scuti. The continuous line is the isochrone corresponding to each cluster. Segments (two per star) represent the possible non-rotating counterparts for each observed star (see details in Sect. 4).

with the angle of inclination, $i$, and the rotation rate, $\omega$. This last dimensionless quantity is defined by

$\omega \equiv \frac{\Omega}{\tilde{\Omega}_{\mathrm{c}}} \quad$ with $\quad \tilde{\Omega}_{\mathrm{c}}^{2} \equiv \frac{8 G M}{27 R_{\mathrm{p}}^{3}}$

where $\Omega$ is the angular rotational velocity of the star; $\tilde{\Omega}_{\mathrm{c}}$ is the angular rotational velocity that a star with the same mass $(M)$ and polar radius $\left(R_{\mathrm{p}}\right)$ would have if the centrifugal force balanced the gravitational attraction at the equator. The nonrotating counterparts are thus computed as a function of the angle of inclination, from $i=90^{\circ}\left(\omega_{\min }\right)$ to $i_{\min }$ obtained for $\omega_{\max }=0.95$.

For each selected $\delta$ Scuti star we compute two associated segments corresponding to corrections assuming an estimation of the error in $V \sin i$ of $\pm 10 \%$. Finally, models at the intersection of segments with the isochrone will be our non-rotating 

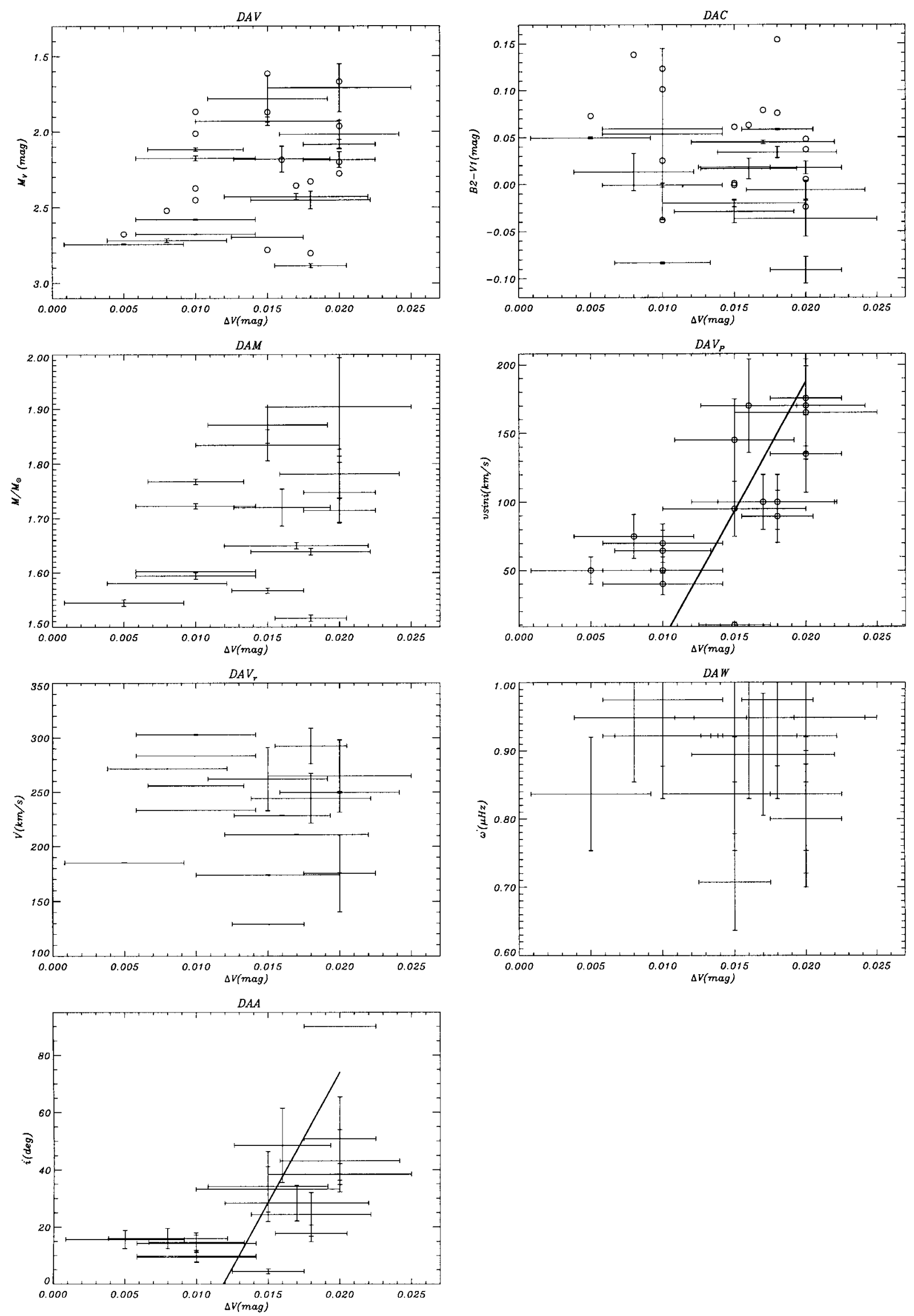

Fig. 3. Correlation diagrams for a set of photometric parameters obtained from the correction for rotation (see Table 6). Circles represent the $\delta$ Scuti stars before correcting for the effect of rotation. Crosses represent the error bars (See Sect. 4 for details). 
Table 3. Correlation between parameters corrected for rotation and the $A_{\text {osc }}$ for our sample of $17 \delta$ Scuti stars. The Spearman Rank Order coefficient is represented by $r_{\mathrm{s}} ; r$ is the linear Pearson coefficient; $\sigma\left(r_{\mathrm{s}}\right)$ $\sigma(r)$ are the errors in $r_{\mathrm{s}}$ and $r$ respectively, and $p$ is a probability of deviation from null-hypothesis.

\begin{tabular}{cccccc}
\hline \hline Parameter & $r_{\mathrm{s}}$ & $\sigma\left(r_{\mathrm{s}}\right)$ & $p\left(r_{\mathrm{s}}\right)$ & $r$ & $\sigma(r)$ \\
\hline$M_{v}$ & 0.4319 & 0.1384 & 0.0834 & 0.4569 & 0.1431 \\
$B_{2}-V_{1}$ & 0.2725 & 0.0691 & 0.2898 & 0.2484 & 0.0571 \\
$i^{\prime}$ & 0.7481 & 0.0447 & 0.0005 & 0.6553 & 0.0447 \\
$V \sin i$ & 0.7663 & 0.0364 & 0.0003 & 0.7122 & 0.0063 \\
$V^{\prime}$ & -0.2016 & 0.0360 & 0.4376 & -0.1810 & 0.0290 \\
$M / M_{\odot}$ & 0.3895 & 0.1251 & 0.1221 & 0.4253 & 0.1367 \\
$\omega^{\prime}$ & -0.1260 & 0.0140 & 0.629 & -0.1223 & 0.0133 \\
\hline
\end{tabular}

counterparts, giving a set of corrected photometric parameters, such as absolute magnitudes $\left(M_{v}\right)$ and $\left(B_{2}-V_{1}\right)$ colour indices. In addition to this, the method gives us the possibility of obtaining an estimation of other stellar parameters such as the angle of inclination of the star, the radial velocity, and the rotation rate, given in Table 6 .

\section{Searching for correlations with the oscillation amplitude}

\subsection{The errors}

We search for correlations between, on one hand, the $A_{\text {osc }}$ parameter, and on the other hand, stellar parameters essentially obtained after correcting for the effect of fast rotation. Here we discuss the error on these parameters.

For $A_{\text {osc }}$, we estimate 0.005 and 0.01 as minimum and maximum typical errors and we calculate error bars assuming a linear relation with the weights listed in Table 5.

Considering the stellar parameters, we have to deal with different error sources: input photometric indexes, $V \sin i$ values, distances, models and calibrations (isochrones), and of course, the method to correct parameters for the effect of rotation.

The photometric input parameters, basically the $m_{V}$ and $B_{2}-V_{1}$, have typical observational errors around $10^{-3}$ magnitudes. This is between 10 and 100 times smaller than the rotation effect found for these parameters (Pérez Hernández et al. 1999; Suárez et al. 2001). The error in the estimation of $V \sin i$ (estimated to $10 \%$ ) is probably a dominant factor here. It can induce errors up to 0.15 and $0.02 \mathrm{mag}$ in $M_{v}$ and $B_{2}-V_{1}$ corrected values respectively. The error on the fit of the isochrones (including those on the distance determination and on the calibration between $B_{2}-V_{1}$ and $T_{\text {eff }}$ ) can be of the same order as that associated with $V \sin i$. However they are expected to be systematic, at least for objects within the same cluster.

The errors associated more specifically with the models and the correction for fast rotation are difficult to estimate, because we lack elements to compare with. Although for $M_{v}$ and $B_{2}-V_{1}$, they are expected to be systematic to an extent, for the rest of the estimated stellar parameters we cannot guarantee it.
Table 4. Linear functional dependence between $V \sin i, i^{\prime}$ and $A_{\text {osc }}$. The linear fit parameters are $a\left(\times 10^{3}\right)$ and $b$ following $y=a A_{\text {osc }}+b$. Their corresponding errors are $\sigma(a)\left(\times 10^{3}\right)$ and $\sigma(b) \cdot \chi^{2}$ represents the minimum Chi-Square of the linear fit and $q$ is a scalar between 0 and 1 giving the probability that a right model would give a value equal or larger than the observed $\chi^{2}$.

\begin{tabular}{ccccccc}
\hline \hline$y$ & $a$ & $\sigma(a)$ & $(b)$ & $\sigma(b)$ & $\chi^{2}$ & $q$ \\
\hline$V \sin i$ & 1.88 & 1.12 & -189.64 & 305.430 & 3.997 & 0.997 \\
$i^{\prime}$ & 9.12 & 5.5 & -108.3 & 156.69 & 4.13 & 0.997 \\
\hline
\end{tabular}

In particular for $i, V$ and $\omega$, we can only claim to obtain rough estimations, which will be indicated with a $\left(^{\prime}\right)$.

We thus consider that the error on $V \sin i$ is representative of the non-systematic errors here, being the most important source of errors when searching for correlations. In addition, other observational aspects such as unknown binarity, differences between the metallicity of the cluster and possible not detected differential extinction inside the cluster, can have an influence.

For corrected parameters in Table 6, the error bars correspond to values obtained using $\pm 10 \%$ in the observed $V \sin i$. In Fig. 3 we present the different diagrams with the parameters corrected for rotation versus $A_{\text {osc }}$, with the error bars for both.

\subsection{The correlation technique}

We computed the Spearman rank-order correlation coefficient, $r_{\mathrm{s}}$. This coefficient is preferred to the classic Pearson one (also computed as a complement) as it is more robust to outliers and does not presuppose a linear relation. For the error made in the correlation coefficient, we use the following expression from standard statistics,

$\sigma\left(r_{\mathrm{s}}\right)^{2}=\frac{\left(1-r_{\mathrm{s}}\right)^{2}}{n-1}\left(1+\frac{11 r_{\mathrm{s}}^{2}}{2 n}\right)+O\left(n^{-3}\right)$

where $n$ is the dimension of the sample. In addition to this, we computed, for the Spearman coefficient, the parameter $p$ which gives the probability of deviation from the null-hypothesis for this coefficient.

On the other hand, for the $r_{\mathrm{s}}$ values indicating a reasonable degree of correlation, we also search for a possible functional dependence. In such cases, we have used an orthogonal distance regression routine (Press et al. 1989) that combines data sets with errors in both variables.

\subsection{Results and discussion}

We have applied the statistical methods described here to all parameters corrected for rotation computed in Sect. 4. In Table 3 the coefficients obtained for correlations between these parameters and the $A_{\text {osc }}$ are listed.

We now analyze the results obtained for the absolute magnitude and the colour index. These two photometric parameters have been generally used in the literature for statistical works on $\delta$ Scuti stars. Particularly, in a multi-correlate analysis made by Antonello et al. (1981), a significant individual correlation between $A_{\mathrm{osc}}$ and $M_{v}$ is found. No strong correlation is found 
Table 5. Photometric data for the $17 \delta$ Scuti stars of the 5 open clusters considered. Different columns represent the star HD (SAO) number, the absolute magnitude, the $B_{2}-V_{1}$ colour index, the oscillation amplitude $A_{\text {osc }}$, the projected velocity $V \sin i$, the spectral type and the class of luminosity, the time observation weight (see Sect. 2) and the cluster they belong to.

\begin{tabular}{ccrccccc}
\hline \hline Star & $\begin{array}{c}M_{v} \\
(\mathrm{mag})\end{array}$ & $\begin{array}{r}B_{2}-V_{1} \\
(\mathrm{mag})\end{array}$ & $\begin{array}{c}A_{\text {osc }}(\mathrm{mag}) \\
\left(\mathrm{km} \mathrm{s}^{-1}\right)\end{array}$ & $\begin{array}{c}V \sin i \\
\text { Sp }\end{array}$ & Weight & Cluster \\
\hline HD 74050 & 1.62 & 0.001 & 0.015 & 145 & A7V & 2 & Praesepe \\
HD 74028 & 1.67 & 0.005 & 0.020 & 165 & A7III & 1 & Praesepe \\
HD 73345 & 1.87 & -0.001 & 0.015 & 95 & F0V & 1 & Praesepe \\
HD 73175 & 1.96 & 0.037 & 0.020 & 170 & A9V & 2 & Praesepe \\
HD 73798 & 2.12 & 0.063 & 0.016 & 170 & F0V & 4 & Praesepe \\
HD 73450 & 2.10 & 0.048 & 0.020 & 135 & A7V & 3 & Praesepe \\
HD 73746 & 2.36 & 0.079 & 0.017 & 110 & A9V & 1 & Praesepe \\
HD 23156 & 2.01 & -0.038 & 0.010 & 65 & A7V & 3 & Pleiades \\
HD 23567 & 2.80 & 0.154 & 0.018 & 90 & A9V & 4 & Pleiades \\
HD 23607 & 2.78 & 0.061 & 0.015 & 10 & A7V & 4 & Pleiades \\
HD 23643 & 2.28 & -0.024 & 0.020 & 175 & A3V & 4 & Pleiades \\
HD 27397 & 2.33 & 0.076 & 0.018 & 100 & F0V & 2 & Hyades \\
HD 27459 & 1.87 & 0.025 & 0.010 & 70 & A9V & 2 & Hyades \\
HD 27628 & 2.45 & 0.101 & 0.010 & 40 & Am & 2 & Hyades \\
HD 107513 & 2.68 & 0.073 & 0.005 & 50 & Am & 2 & Coma Ber \\
SAO 38754 & 2.52 & 0.138 & 0.008 & 75 & F0IV & 2 & $\alpha$-Persei \\
SAO 38788 & 2.37 & 0.123 & 0.010 & 50 & A8V & 2 & $\alpha$-Persei \\
\hline
\end{tabular}

here for $M_{v}$ or for $B_{2}-V_{1}$, however we notice that the correlation coefficient is approximately two times better for corrected values of $M_{v}$ than for non-corrected ones $\left(r_{\mathrm{s}}=0.26\right)$. They are of the same order in the case of $B_{2}-V_{1}\left(r_{\mathrm{s}} \sim 0.25\right)$. We thus conclude, following Suárez et al. (2001), that it is worthwhile to take into account such a correction in this kind of study. We attribute the low value obtained for the coefficient in the case of the magnitude to the fact that our sample is still too limited.

A less classical parameter for such studies is the projected rotational velocity, $V \sin i$, for which an unexpected significant correlation is found $\left(r_{\mathrm{s}}=0.76\right)$. This parameter has been considered before in Li \& Michel (1999), however no correlation was calculated. This striking result has two possible explanations: a physical effect (linked to $V^{\prime}$ or maybe rather $\omega^{\prime}$ ) and/or a geometric effect (associated with $i^{\prime}$ ). Making use of the different stellar parameters we have access to, we tried to go further to understand the origin of this correlation. We can notice that there is no significant correlation for the $V^{\prime}$ parameter or for the $\omega^{\prime}$ parameter.

On the other hand, for $i^{\prime}$, another significant correlation is found ( $\left.r_{\mathrm{s}}=0.75\right)$ such that, the higher the value of $A_{\text {osc }}$ the larger $i^{\prime}$. These results suggest that the correlation observed for $V \sin i$ has a pure geometric origin. At this point, we tried to calculate if a possible bias could be induced by the method used to correct parameters for rotation. We can only figure out a possible influence of our limited sample of stars. In addition, the correlation found between $V \sin i$ and $A_{\text {osc }}$ does not involve the correction method.

In the framework of the classical description of the oscillations, which attributes to each mode a given spherical harmonic, the preceding result would first confirm that the variation of flux observed in $\delta$ Scuti light curves are not dominated by the radial modes, whose visibility coefficients are independent of $i^{\prime}$. This is in agreement with the results obtained by Hernández et al. (1998) who showed that they could not find more than two radial modes per star, in oscillation spectra, for a group of selected stars in the Praesepe cluster.

The $A_{\text {osc }}$ value is considered as the maximum constructive interference of the observed modes. Then the present results would suggest that, at least, some modes with visibility coefficients increasing with $i^{\prime}$ are favored in the $\delta$ Scuti star oscillations compared with other modes.

As can be seen in Pesnell (1985), for instance, modes corresponding to this description are the sectoral modes $(m= \pm \ell)$, as opposed to the zonal modes $((\ell=1, m=0),(\ell=2, m=0))$ whose visibility coefficient is low for $i \rightarrow 0$, and $(\ell=2, m=$ \pm 1 ) whose visibility coefficient is low for $i \rightarrow 0$ and for $i \rightarrow 90$. In this context, our results would suggest that the $\delta$ Scuti oscillations are dominated, at least to some extent, by modes with $m= \pm \ell$. If confirmed, this interpretation would constitute an important new element in the problem of mode identification of $\delta$ Scuti stars.

However, we want to stress that the classical spherical harmonics system might not be the best context to interpret this result. It is well known now that $\delta$ Scuti stars are fast rotators (this is confirmed here where, for our sample of $\delta$ Scuti stars, large $V$ values are found) and that the effect of rotation affects their structure to a point where the description in terms of spherical harmonics might become questionable. An alternative representation is being developed by Lignières (Lignières 2001) by means of a non-perturbative numerical method. Their results obtained for homogeneous ellipsoids show modes with 
Table 6. Non-rotating associate models for the $\delta$ Scuti stars in the 5 open clusters considered. The different columns represent the range of values obtained from the correction for the effect of rotation (see Sect. 4) for: $M_{v}$ the absolute magnitude; $B_{2}-V_{1}$ the colour index; $M$ the mass (in $M_{\odot}$ ); $R$ the radius (in $R_{\odot}$ ); $T_{\text {eff }}$ the effective temperature (log, in $\mathrm{K}$ ); $L$ the luminosity (in $\left.L_{\odot}\right) ; \omega^{\prime}$ the rotation rate; $v_{\text {rot' }}$ the rotation frequency $(\mu \mathrm{Hz}) ; i^{\prime}$ the angle of inclination (deg), and finally $g$ the gravity $\left(\log\right.$, in $\left.\mathrm{cm} \mathrm{s}^{2}\right)$.

\begin{tabular}{|c|c|c|c|c|c|}
\hline Star & $\begin{array}{c}M_{v} \\
L / L_{\odot}\end{array}$ & $\begin{array}{c}B_{2}-V_{1} \\
\omega^{\prime}\end{array}$ & $\begin{array}{c}M / M_{\odot} \\
v_{\mathrm{rot}^{\prime}}\end{array}$ & $\begin{array}{c}R / R_{\odot} \\
i^{\prime}\end{array}$ & $\begin{array}{c}\log T_{\text {eff }} \\
\log g\end{array}$ \\
\hline \multirow[t]{2}{*}{ HD 74050} & $1.705-1.859$ & $-0.035-(-0.023)$ & $1.89-1.86$ & $2.09-2.04$ & $3.90-3.89$ \\
\hline & $15.65-14.46$ & $0.90-0.90$ & $25.08-25.82$ & $40.28-28.04$ & $4.07-4.08$ \\
\hline \multirow[t]{2}{*}{ HD 74028} & $1.631-1.790$ & $-0.046-(-0.027)$ & $1.95-1.86$ & $2.19-2.05$ & $3.90-3.89$ \\
\hline & $18.06-14.65$ & $0.90-0.90$ & $23.64-25.68$ & $40.28-36.59$ & $4.04-4.08$ \\
\hline \multirow[t]{2}{*}{ HD 73345} & $1.916-1.944$ & $-0.022-(-0.018)$ & $1.85-1.82$ & $2.03-1.99$ & $3.89-3.89$ \\
\hline & $14.27-13.53$ & $0.70-0.70$ & $25.77-26.19$ & $37.16-29.21$ & $4.01-4.09$ \\
\hline \multirow[t]{2}{*}{ HD 73175} & $1.971-2.063$ & $-0.011-(-0.001)$ & $1.80-1.76$ & $1.97-1.91$ & $3.89-3.88$ \\
\hline & $12.83-11.52$ & $0.90-0.90$ & $26.88-27.83$ & $48.57-37.69$ & $4.11-4.12$ \\
\hline \multirow[t]{2}{*}{ HD 73798} & $2.139-2.225$ & $0.011-0.022$ & $1.74-1.70$ & $1.88-1.84$ & $3.88-3.88$ \\
\hline & $10.91-10.05$ & $0.85-0.85$ & $28.25-28.91$ & $54.98-42.02$ & $4.13-4.14$ \\
\hline \multirow[t]{2}{*}{ HD 73450} & $2.160-2.213$ & $0.014-0.021$ & $1.73-1.70$ & $1.86-1.84$ & $3.88-3.88$ \\
\hline & $10.62-10.05$ & $0.70-0.70$ & $28.35-28.78$ & $58.15-43.56$ & $4.13-4.14$ \\
\hline \multirow[t]{2}{*}{ HD 73746} & $2.420-2.440$ & $0.044-0.046$ & $1.65-1.65$ & $1.75-1.74$ & $3.87-3.87$ \\
\hline & $8.48-8.32$ & $0.80-0.80$ & $30.51-30.69$ & $31.45-25.24$ & $4.17-4.17$ \\
\hline \multirow[t]{2}{*}{ HD 23156} & $2.112-2.123$ & $-0.084-(-0.083)$ & $1.77-1.76$ & $1.52-1.52$ & $3.93-3.94$ \\
\hline & $11.86-11.72$ & $0.85-0.85$ & $39.09-39.15$ & $16.34-12.89$ & $4.32-4.32$ \\
\hline \multirow[t]{2}{*}{ HD 23567} & $2.879-2.892$ & $0.059-0.058$ & $1.52-1.51$ & $1.41-1.41$ & $3.88-3.87$ \\
\hline & $5.57-5.46$ & $0.95-0.95$ & $40.63-40.64$ & $19.22-16.34$ & $4.32-4.31$ \\
\hline \multirow[t]{2}{*}{ HD 23607} & $2.698-2.698$ & $0.018-0.018$ & $1.57-1.56$ & $1.43-1.43$ & $3.89-3.88$ \\
\hline & $6.63-6.50$ & $0.50-0.50$ & $39.87-39.93$ & $4.88-4.00$ & $4.32-4.32$ \\
\hline \multirow[t]{2}{*}{ HD 23643} & $2.068-2.099$ & $-0.098-(-0.086)$ & $1.77-1.72$ & $1.523-1.49$ & $3.94-3.93$ \\
\hline & $12.00-10.63$ & $0.69-0.59$ & $38.89-38.25$ & $90.00-90.00$ & $4.32-4.32$ \\
\hline \multirow[t]{2}{*}{ HD 27397} & $2.423-2.481$ & $0.037-0.031$ & $1.64-1.64$ & $2.029-2.02$ & $3.87-3.87$ \\
\hline & $8.27-8.11$ & $0.85-0.85$ & $24.44-24.48$ & $28.18-20.59$ & $4.19-4.19$ \\
\hline \multirow[t]{2}{*}{ HD 27459} & $2.168-2.184$ & $-0.002-0.000$ & $1.72-1.72$ & $2.152-2.15$ & $3.89-3.88$ \\
\hline & $10.55-10.68$ & $0.95-0.95$ & $22.98-22.96$ & $15.77-12.84$ & $4.16-4.16$ \\
\hline \multirow[t]{2}{*}{ HD 27628} & $2.579-2.582$ & $0.059-0.059$ & $1.60-1.60$ & $2.072-2.07$ & $3.87-3.87$ \\
\hline & $7.20-7.21$ & $0.85-0.85$ & 23.39-23.39 & $10.86-8.87$ & $4.21-4.20$ \\
\hline \multirow[t]{2}{*}{ HD 107513} & $2.743-2.746$ & $0.050-0.049$ & $1.55-1.54$ & $1.499-1.75$ & $3.87-3.87$ \\
\hline & $6.30-6.17$ & $0.70-0.70$ & $37.18-29.44$ & $17.29-14.07$ & $4.27-4.27$ \\
\hline \multirow[t]{2}{*}{ SAO 38754} & $2.713-2.726$ & $0.023-0.003$ & $1.58-1.580$ & $1.455-1.45$ & $3.88-3.88$ \\
\hline & $6.56-6.55$ & $0.90-0.90$ & $39.54-39.58$ & $17.79-14.28$ & $4.31-4.31$ \\
\hline \multirow[t]{2}{*}{ SAO 38788} & $2.678-2.676$ & $0.099-0.008$ & $1.59-1.591$ & $1.461-1.45$ & $3.89-3.88$ \\
\hline & $6.93-6.80$ & $0.95-0.95$ & $39.58-39.79$ & $10.45-08.55$ & $4.31-4.31$ \\
\hline
\end{tabular}

geometric horizontal features very different from classical spherical harmonics. These results are preliminary and have to be applied to models closer to stellar internal structure, but they might be indicative of relevant geometric effects.

For projected velocity and $i^{\prime}$, we have also searched for a functional dependence. In Table 4 their linear fit parameters are listed. We have to keep in mind that the coefficients of these linear fits might be sensitive to eventual systematic errors, which were neglected when searching for correlations (see Sect. 5). While good confidence values $(q)$ are obtained, we can appreciate from values of $\chi^{2}$ that scatter is important as well as errors in fit coefficients. This situation can be improved by increasing the number of target stars.

Finally, no correlation is found for the mass.

\section{Conclusions}

For a sample of $17 \delta$ Scuti stars, we report here the results of a search for correlations between the oscillation amplitude and a set of estimated fundamental parameters. 
In this process we confirm the interest of applying a correction for fast rotation, as suggested by Suárez et al. (2001) for this kind of study.

We found a significant correlation coefficient for the projected velocity $(V \sin i)$ and show that it is due to the strong correlation between the oscillation amplitude and an estimation of the angle of inclination of the star, $i^{\prime}$. We observe that the oscillation amplitudes increase with $i^{\prime}$. This striking result is discussed in terms of the visibility of modes. In the context of spherical harmonics, this suggests that the oscillations of $\delta$ Scuti stars would follow a selection rule in favor of sectoral modes (i.e. $m= \pm \ell$ modes).

An alternative interpretation might appear with future developments of the work initiated by Lignières (Lignières 2001).

A future increase in our sample of $\delta$ Scuti stars in clusters would help to refine the present results.

\section{References}

Antonello, E., Fracassini, M., \& Pastori, L. 1981, Ap\&SS, 78, 435 Breger, M. 2000, in Delta Scuti and Related Wtars, Reference Handbook and Proceedings of the 6th Vienna Workshop in Astrophysics, held in Vienna, Austria, 4-7 August, 1999, ed. M. Breger, \& M. Montgomery (San Francisco: ASP) ISBN: 1-58381041-2, 2000, ASP Conf. Ser., 210, 3

de Bruijne, J. H. J., Hoogerwerf, R., \& de Zeeuw, P. T. 2001, A\&A, 367,111

Grenon, M. 2000, HIPPARCOS and the Luminosity Calibration of the Nearer Stars, 24th meeting of the IAU, Joint Discussion 13, August 2000, Manchester, UK, 13

Handler, G. 2000, in The Impact of Large-Scale Surveys on Pulsating Star Research, ASP Conf. Ser., 203, IAU Colloq., 176, 408

Hernández, M. M., Pérez Hernández, F., Michel, E., et al. 1998, A\&A, 338,511
Iglesias, C. A., \& Rogers, F. J. 1996, ApJ, 464, 943

Kunzli, M., North, P., Kurucz, R. L., \& Nicolet, B. 1997, A\&AS, 122, 51

Li, Z. P., \& Michel, E. 1999, A\&A, 344, L41

Lignières, F. 2001, in SF2A-2001: Semaine de l'Astrophysique Française, E98

Michel, E., Hernández, M. M., Houdek, G., et al. 1999, A\&A, 342, 153

Morel, P. 1997, A\&AS, 124, 597

Nicolet, B. 1981, A\&A, 104, 185

Pérez Hernández, F., Claret, A., Hernández, M. M., \& Michel, E. 1999, A\&A, 346, 586

Perryman, M. A. C., Brown, A. G. A., Lebreton, Y., et al. 1998, A\&A, 331,81

Pesnell, W. D. 1985, ApJ, 292, 238

Pinsonneault, M. H., Stauffer, J., Soderblom, D. R., King, J. R., \& Hanson, R. B. 1998, ApJ, 504, 170

Press, W. H., Flannery, B. P., Teukolsky, S. A., \& Vetterling, W. T. 1989, Numerical recipes in Pascal. The art of scientific computing (Cambridge: University Press, -c1989)

Robichon, N., Arenou, F., Mermilliod, J.-C., \& Turon, C. 1999, A\&A, 345,471

Rufener, F. 1999, VizieR Online Data Catalog, 2169, 0

Schaller, G., Schaerer, D., Meynet, G., \& Maeder, A. 1992, A\&AS, 96, 269

Schmidt-Kaler, T. 1982, Bulletin d'Information du Centre de Données Stellaires, 23, 2

Stello, D., \& Nissen, P. E. 2001, A\&A, 374, 105

Suárez, J.-C., Michel, E., Fox, L., et al. 2001, Proceedings of the SOHO 10/GONG 2000 Workshop: Tenerife, Spain, ed. A. Wilson, ESA SP-464 (Noordwijk: ESA Publications Division), ISBN 92-9092-697-X, 461

Uesugi, A., \& Fukuda, I. 1982, Catalogue of stellar rotational velocities (revised) (Kyoto: University of Kyoto, Departement of Astronomy, 1982, Rev.ed.) 